\title{
Fatigue is not a necessary stimulus for strength gains during resistance training
}

\author{
J P Folland, C S Irish, J C Roberts, J E Tarr, D A Jones
}

Br J Sports Med 2002;36:370-374

See end of article for authors' affiliations

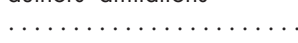

Correspondence to:

Dr Folland, Chelsea School

Research Centre, University of Brighton, Gaudick Road, Eastbourne BN20 7SP, UK j.folland@bton.ac.uk

Accepted

6 February 2002

\begin{abstract}
Background: High resistance training enhances muscular strength, and recent work has suggested an important role for metabolite accumulation in this process.

Objective: To investigate the role of fatigue and metabolite accumulation in strength gains by comparing highly fatiguing and non-fatiguing isotonic training protocols.

Methods: Twenty three healthy adults (18-29 years of age; eight women) were assigned to either a high fatigue protocol (HF: four sets of 10 repetitions with 30 seconds rest between sets) to maximise metabolic stress or a low fatigue protocol (LF: 40 repetitions with 30 seconds between each repetition) to minimise changes. Subjects lifted on average $73 \%$ of their 1 repetition maximum through the full range of knee extension with both legs, three times a week. Quadriceps isometric strength of each leg was measured at a knee joint angle of $1.57 \mathrm{rad}\left(90^{\circ}\right)$, and a Cybex 340 isokinetic dynamometer was used to measure the angle-torque and torque-velocity relations of the non-dominant leg.

Results: At the mid-point of the training, the HF group had 50\% greater gains in isometric strength, although this was not significant (4.5 weeks: HF, $13.3(4.4) \%$; LF, $8.9(3.6) \%)$. This rate of increase was not sustained by the HF group, and after nine weeks of training all the strength measurements showed similar improvements for both groups (isometric strength: HF, 18.2 (3.9)\%; LF, 14.5 (4.0)\%). The strength gains were limited to the longer muscle lengths despite training over the full range of movement.

Conclusions: Fatigue and metabolite accumulation do not appear to be critical stimuli for strength gain, and resistance training can be effective without the severe discomfort and acute physical effort associated with fatiguing contractions.
\end{abstract}

$\mathrm{H}$ igh resistance training is known to enhance muscular strength and promote hypertrophy, but the physiological link between the activity and the increased accumulation of contractile proteins required for these adaptations has yet to be elucidated. Much has been written about the merits of different training regimens and it is important from both practical and theoretical perspectives to establish the most effective means of increasing strength. It is clear that high force muscle actions are required to increase strength but beyond this there is considerable uncertainty. ${ }^{1-3}$

There has been extensive discussion on the benefits of mechanical and metabolic stimuli for strength gains, which have been investigated by comparing eccentric and concentric muscle actions. With one exception, ${ }^{4}$ studies that have compared purely eccentric and concentric training have failed to show eccentric work to produce greater isometric strength gains or hypertrophy. ${ }^{5-8}$

High force concentric and isometric contractions involve considerable metabolic flux at a time when the blood supply is occluded by the high force contractions. Metabolic byproducts $\left(\mathrm{H}^{+}\right.$, lactate, $\left.\mathrm{P}_{\mathrm{i}}, \mathrm{Cr}, \mathrm{K}^{+}\right)$accumulate both inside and outside muscle fibres, and the build up of these metabolites is associated with fatigue as well as pain and discomfort. Recent work has suggested that metabolite accumulation during high resistance work may be the primary stimulus for gains in strength and muscle hypertrophy. ${ }^{6-11}$

Shinohara $e t ~ a l^{11}$ reported that training under ischaemic conditions almost doubled the increase in isometric strength $(26 \% v 14 \%)$ after only four weeks of training at $40 \%$ maximal voluntary contractile force. They concluded that metabolite accumulation may be more important than high forces for optimal strength training. Schott $e t$ a $l^{10}$ compared intermittent and sustained isometric contractions, with the two protocols involving the same load and duration of loading. The sustained contractions caused higher metabolite accumulation and resulted in significantly greater gains (about 80\%) in isometric strength. In contrast, a later study from the same group found no advantage of high metabolite accumulation after six months of isometric training of older subjects. ${ }^{12}$

Rooney et al ${ }^{9}$ applied the possible advantage of metabolite accumulation to normal isotonic strength training. They compared a "no rest" cadence with one with 30 seconds rest in between every repetition. After six weeks of training the dynamic strength on the training exercise ( 1 repetition maximum (IRM)) increased significantly more in the "no rest" condition. However, in terms of isometric strength, there was no significant difference $(22.1 \vee 19.8 \%)$.

Hence there is recent evidence in favour of a metabolic stimulus for muscle strength gains during resistance training, and these reports suggest that the pain and discomfort associated with fatigue and high metabolite accumulation are essential to optimise strength training. We sought to elucidate further the role of fatigue and metabolite accumulation during nine weeks of conventional isotonic strength training. A preliminary account of these findings has been published in abstract form. ${ }^{13}$

\section{METHODS}

\section{Subjects}

At the start of the study, 30 volunteers were paired according to isometric strength, body mass, and sex, and assigned to either a high fatigue (HF) or low fatigue (LF) training protocol. Twenty three subjects completed the study (table 1), with subject drop out randomly distributed between the groups. Most subjects were recreationally active, but none had a history of leg strength training or knee pathology. Subjects were asked to maintain their habitual levels of activity 


$\begin{aligned} & \text { Table } 1 \\
& \text { groups }\end{aligned}$
\begin{tabular}{llllllll} 
& Subject characteristics for the high fatigue (HF) and low fatigue (LF) training \\
& Men & Women & Age (years) & Mass (kg) & Stature (m) & $\begin{array}{l}\text { Physical activity } \\
\text { (h/week) }\end{array}$ \\
\hline HF & 8 & 4 & $22(2)$ & $70(3)$ & $1.81(0.09)$ & $2.3(1.5)$ \\
LF & 7 & 4 & $20(1)$ & $68(7)$ & $1.76(0.10)$ & $2.0(1.2)$ \\
\hline
\end{tabular}

Values are mean (SD).

throughout the study period. The study protocol was approved by the local ethics committee, and subjects gave their informed consent.

\section{Training}

The training consisted of three sessions a week (Monday, Wednesday, and Friday) for nine weeks, and was conducted with one to one investigator to subject supervision. Subjects trained the quadriceps femoris muscle group bilaterally on a variable resistance leg extension machine (VR Universal, Cedar Rapids, Iowa, USA), through a range of angles from 2.09 to $0.52 \mathrm{rad}$ of knee flexion $\left(115-30^{\circ}\right)$. The maximum load that could be lifted just once (IRM) was measured to the nearest $2.0 \mathrm{~kg}$ and reassessed every week. The training load for both protocols was specified as $75 \%$ of $1 \mathrm{RM}$, and the total number of repetitions was the same for both protocols. The two training protocols were designed to differentiate as much as possible for fatigue and metabolite accumulation.

\section{High fatigue training (HF)}

Subjects completed four sets of 10 repetitions with 30 seconds of rest between sets. They were instructed to perform the contractions in each set continuously in a smooth controlled manner, lowering the weight fully and lifting it through the full range. To ensure maximum and demonstrable fatigue, this protocol was designed to be so severe that it was impossible for the subjects to complete all the contractions at the prescribed load (75\% 1RM). When they could no longer lift the prescribed load through at least two thirds of its range, the load was reduced and the training continued immediately. The training load was typically reduced two or three times in a training session.

\section{Low fatigue training (LF)}

Subjects performed l repetition every 30 seconds to minimise fatigue and metabolite accumulation. Forty repetitions were completed during each training session, and it was never necessary to reduce the training load for any subject following this protocol. Again, the subjects were instructed to perform each repetition in a smooth controlled manner lifting the weight through the whole range.

\section{Strength testing}

Quadriceps muscle strength was assessed on four occasions before the training, each one week apart, allowing the subjects to become familiar with the testing environment and procedures. Values before training were designated as the average of the third and fourth of these baseline measurements. Further strength tests were carried at the mid point of the training ( 4.5 weeks) and after nine weeks of training. On each testing occasion, isometric strength at $1.57 \mathrm{rad}$ and angle-torque and torque-velocity relations were measured.

\section{Isometric strength}

Maximum isometric strength was measured using a conventional strength testing chair. ${ }^{14}$ This involved three maximal efforts of knee extension of at least two seconds duration with 30 seconds of rest between each contraction. The two legs were tested separately at a joint angle of $1.57 \mathrm{rad}\left(90^{\circ}\right)$. This measurement was used as the criterion strength measurement because of its high reproducibility (during the four baseline measurements, the coefficient of variation was $4.9 \%$ ).

A Cybex 340 isokinetic dynamometer (Lumex Inc, Ronkokama, New York, USA) was used to assess the angle-torque and torque-velocity relations of the nondominant leg. The order of testing angles and velocities was selected randomly for each subject and maintained on successive testing occasions.

\section{Angle-torque relation}

Isometric torque was measured at $0.26 \mathrm{rad}\left(15^{\circ}\right)$ intervals from $1.83 \mathrm{rad}\left(105^{\circ}\right)$ to $0.52 \mathrm{rad}\left(30^{\circ}\right)$ of knee flexion ( $\mathrm{six}$ angles in total). Subjects made a practice effort followed by a maximal voluntary contraction of three seconds duration at each angle, with 60 seconds rest between each angle.

\section{Torque-velocity relation}

Peak isokinetic torque, irrespective of angle, was assessed at angular velocities of $0.78,1.57,2.35,3.14,4.19$, and $5.24 \mathrm{rad} / \mathrm{s}$ $(45,90,135,180,240$, and $300 \%$ s $)$. Subjects performed four practice trials, before three maximal efforts were recorded at each angular velocity. The range of motion for the isokinetic measurements was from $2.09 \mathrm{rad}\left(120^{\circ}\right)$ to $0.26 \mathrm{rad}\left(15^{\circ}\right)$ of knee flexion, and there was 60 seconds rest between tests at each angular velocity.

\section{Statistical analysis}

Mean (SEM) was calculated for each variable. Analysis of variance was used to examine changes in absolute strength, and Students' $t$ tests were used to investigate differences between groups.

\section{RESULTS}

\section{Training}

Both training groups completed a mean of 24 training sessions (range 21-27) in the nine week period. The HF protocol produced severe muscle soreness in the first week of training, but this was not the case for the LF protocol. The mean IRM increased significantly $(\mathrm{p}<0.05)$ for both groups, from 85 to

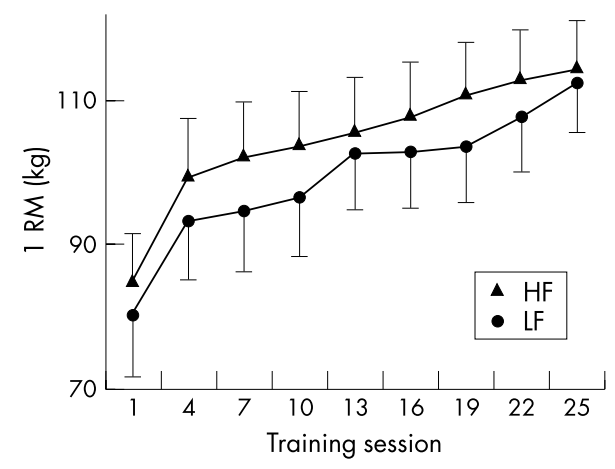

Figure 1 Increase in the 1 repetition maximum (1RM) for the high fatigue (HF) and low fatigue (LF) training groups. Values are mean (SD). 
Table 2 Isometric strength (N) before, midway through, and after training

\begin{tabular}{|c|c|c|c|}
\hline & Before & Mid & After \\
\hline $\begin{array}{l}\text { HF } \\
\text { LF }\end{array}$ & $\begin{array}{l}515(37) \\
505(38)\end{array}$ & $\begin{array}{l}598(44)^{* *} \\
551(47)^{*}\end{array}$ & $\begin{array}{l}609(48)^{* *} \\
576(44)^{* *}\end{array}$ \\
\hline \multicolumn{4}{|c|}{$\begin{array}{l}\text { Values are mean (SEM). } \\
{ }^{*} p<0.01,{ }^{* *} p<0.001 \text { compared with isometric strength before } \\
\text { training. } \\
\text { HF, High fatigue; LF, low fatigue. }\end{array}$} \\
\hline
\end{tabular}

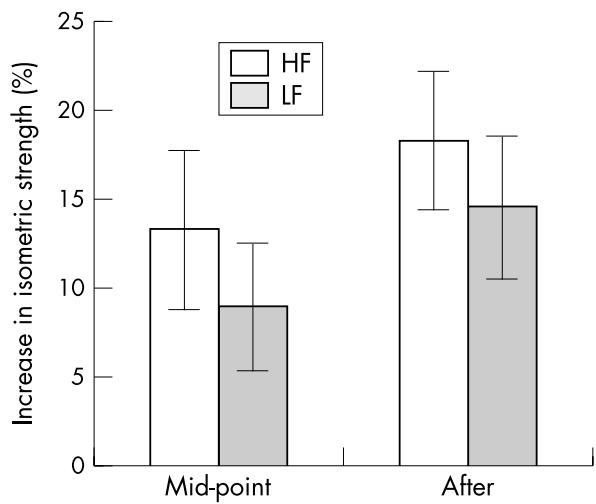

Figure 2 Percentage increase in isometric strength at the mid-point and after training for high fatigue (HF) and low fatigue (LF) training protocols. Values are mean (SEM).

$114 \mathrm{~kg}$ for HF (34\%) and from 80 to $112 \mathrm{~kg}$ for LF (40\%, fig 1 ). There was no significant difference between the gains in lifting strength (1RM) of the two groups after 4.5 weeks ( $p=$ $0.46)$ or nine weeks $(p=0.26)$ of training. The HF group was typically unable to lift 75\% IRM throughout each session and trained at a mean load of $71.8 \% 1 \mathrm{RM}$.

\section{Isometric strength}

The two groups started the training with similar levels of isometric strength (table 2). At the mid-point (4.5 weeks) and after the training (nine weeks) both groups were significantly stronger than before training $(\mathrm{p}<0.01)$. After 4.5 weeks, the mean increase in strength for the HF group was more than $50 \%$ greater than that for the LF group, although this difference was not significant (HF, 13.3 (3.4)\%; LF, 8.9 (2.6)\%; $\mathrm{p}=0.26$ ). There was a large variation in response to training after both 4.5 and nine weeks. At the end of the training, however, the mean increases in strength of the two groups were similar (HF, 18.2 (3.9)\%, range 6 to $43 \%$; LF, 14.5 (4.0)\%, range -5 to $35 \%$; $=0.22$; fig 2 ). This was equivalent to increases of $0.77 \%$ and $0.61 \%$ per training session for HF and LF training respectively.

No significant relations were found between the increase in isometric force and the number of training sessions attended, body mass, activity levels, or strength before training.

\section{Angle-torque}

Strength gains were not uniform along the angle-torque curve (fig 3). There were significant increases in strength at only the longer muscle lengths for both types of training $(1.83,1.57$, and $1.31 \mathrm{rad}$ of flexion, $\mathrm{p}<0.05)$. No significant differences were found between the training groups.

\section{Torque-velocity}

Significant increases in isokinetic strength were found at most velocities for both training groups (fig 4). There was a non-significant tendency towards greater high velocity strength gains in the LF group ( $<<0.10$ at 4.19 and $5.24 \mathrm{rad} / \mathrm{s})$.

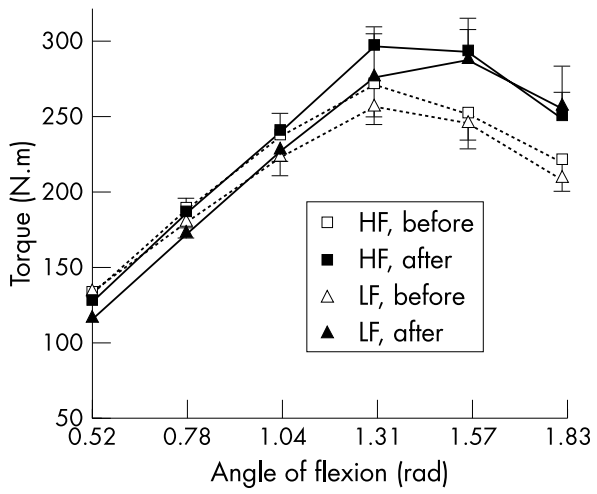

Figure 3 Changes in the angle-torque relation for the high fatigue (HF) and low fatigue (LF) protocol before and after training. Values are mean (SEM).

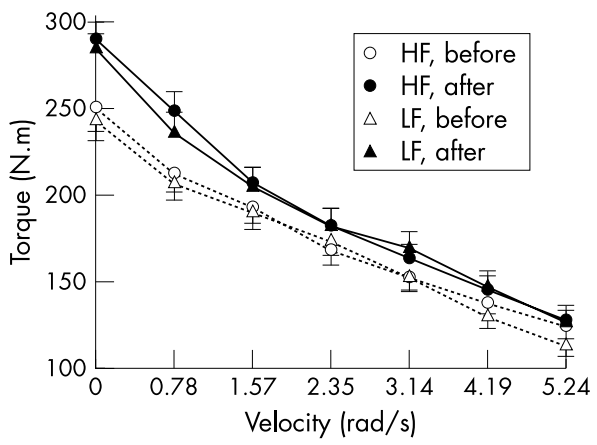

Figure 4 Changes in the concentric torque-velocity relation with the high fatigue (HF) and low fatigue (LF) protocol before and after training. Values are mean (SEM).

\section{DISCUSSION}

The HF and LF training protocols produced similar gains in strength for all of the strength measures (IRM, isometric strength, and isokinetic strength) after 4.5 and nine weeks of training. HF training increased isometric strength rapidly in the first half of the study, but this rate of increase was not sustained. The LF group gained strength more steadily throughout the whole study. Diminishing returns following a rapid initial rise in strength have been widely observed in high resistance training regimens of 8-20 weeks, ${ }^{15}$ and this was also the case for both groups in this investigation.

The main finding of this study was the similarity in the effects of HF and LF training. The greatest disparity between the two training protocols was after 4.5 weeks of training (HF, $13.3 \%$; LF, $8.9 \%$ ). However, this difference was not significant, and therefore our data are at odds with previous short duration studies that have found significantly greater increases in strength with metabolite accumulation after only four to six weeks of training. ${ }^{91}$ During the first week of training, the HF group experienced severe muscle soreness, which is indicative of muscle damage, but this was not the case for the LF group and surprisingly has not been reported by similar investigations of HF training. Muscle damage can cause a strength decrement for days and even weeks, ${ }^{16}{ }^{17}$ and, in theory, this could have attenuated the strength gains and any advantage of HF training. However, we have previously investigated the influence of a more acute bout of muscle damage during the initial stages of a strength training programme and found that this did not influence isometric strength gains for more than two weeks. ${ }^{18}$ Therefore differences in the exact methodology of strength training and the mode of strength measurements recorded would seem to account for these contradictory findings. 
After nine weeks of training, the two training protocols resulted in similar increases in strength $(18.2(3.9) \% v 14.5$ $(4.0) \%$ ). NMR spectroscopy would have been a worthwhile additional measurement to validate the difference in fatigue and metabolite accumulation between the two protocols used. However, we believe that, in practice, it would be difficult to achieve a greater discrepancy in fatigue with volunteer subjects during high resistance training. To ensure maximal and demonstrable fatigue with the HF protocol, this training regimen was designed so that subjects were unable to lift the prescribed load throughout each training session. This inevitably caused a difference in the average load lifted by the two training groups $(71.8 \% \vee 75 \%)$ which may have compromised any effect of fatigue/metabolite accumulation. Despite the use of a lighter load, HF training produced similar gains in strength, and an optimal combination of load and fatigue may be advantageous. Alternatively, it may be possible to complete the LF protocol with a training load higher than $75 \% 1 \mathrm{RM}$ and this may produce greater strength gains than we observed.

Our observations suggest that high fatigue is not an essential or primary stimulus for gains in strength. Low fatigue strength training designed to minimise metabolite accumulation produced significant increases in strength that were of a similar magnitude to training designed to maximise fatigue/ metabolite accumulation. This suggests that significant and comparable strength gains can be achieved with training that involves a low level of discomfort and physical effort. This finding is contrary to two longer studies by Rutherford and colleagues, who found metabolite accumulation to be of significant benefit to strength gains, ${ }^{6}{ }^{10}$ although a six month study by the same group, using a protocol identical with that of one of the previous investigations, found no advantage to high metabolite accumulation. ${ }^{12}$

To address a specific question on the importance of fatigue, we used two rather extreme training protocols. The isometric strength gains of $0.77 \%$ and $0.61 \%$ per training session for HF and LF respectively are similar to gains of $0.61-0.75 \%$ per training session reported by comparable isotonic training studies. ${ }^{6}{ }^{19} 20$ All of these studies used rest intervals between the two extremes of our study, and it would appear therefore that strength gains are not particularly sensitive to the degree of metabolic recovery afforded.

This study had an even wider individual variation in the training response than previously documented, ${ }^{510}$ and there is evidence for a genetic component underlying this variation. ${ }^{21}{ }^{22}$ Such large heterogeneity of sample populations questions the use of intersubject designs in the comparison of training models, and we recommend closer consideration of intrasubject designs when comparing training models, despite the clear problems of cross over effects.

The gains in strength were at long muscle lengths, with little change in strength at the shortest lengths (fig 3). As the training involved the same relative loading of the muscle through the full range of movement-that is, in relation to the angle-torque relation-proportional increases would be expected at all muscle lengths. ${ }^{23}$ Both groups displayed a slight increase in the tension optima after training, which implies a shift of the whole angle-torque relation to the right as a consequence of an increase in muscle length.

Both forms of training significantly increased isokinetic strength at almost all the measured velocities (fig 4). Several reports suggest that strength gains are specific to the velocity of training, ${ }^{24}{ }^{25}$ but our data indicate gains in strength at all movement speeds.

In conclusion, low fatigue strength training designed to minimise metabolic changes produced significant increases in strength comparable to those of high fatigue training designed to maximise metabolite accumulation. Fatigue and metabolite accumulation do not appear to be critical stimuli for strength gains, and resistance training can be effective without severe discomfort and acute physical effort.

\section{Take home message}

Strength training does not need to involve severe discomfort and fatigue to produce significant gains in strength. Effective strength training can be performed with large rest periods, which minimise fatigue and discomfort.

\section{Authors' affiliations}

J P Folland, Chelsea School Research Centre, University of Brighton, Eastbourne, UK

C S Irish, J C Roberts, J E Tarr, D A Jones, School of Sport \& Exercise Sciences, University of Birmingham, Birmingham, UK

\section{REFERENCES}

1 Atha J. Strengthening muscle. Exerc Sport Sci Rev 1981;9:1-73.

2 McDonagh MJN, Davies CTM. Adaptive response of mammalian skeletal muscle to exercise with high loads. Eur J Appl Physiol 1984:52:139-55

3 Jones DA, Rutherford OM, Parker DF. Physiological changes in skeletal muscle as a result of strength training. Q J Exp Physiol 1989;74:233-56. 4 Hortobagyi T, Hill JP, Houmard JA, et al. Adaptive responses to muscle lengthening and shortening in humans. J Appl Physiol 1996;80:765-72.

5 Jones DA, Rutherford OM. Human muscle strength training: the effects of three different training regimes and the nature of the resultant changes. $J$ Physiol (Lond) 1987;391:1-11

6 Carey Smith R, Rutherford OM. The role of metabolites in strength training. I. A comparison of eccentric and concentric contractions. Eur J Appl Physiol 1995;71:332-6.

7 Higbie EJ, Cureton KJ, Warren GL III, et al. Effects of concentric and eccentric training on muscle strength, cross-sectional area, and neural activation. J Appl Physiol 1996;81:2173-81.

8 Godard M, Wygand J, Carpinelli R, et al. The effects of accentuated eccentric resistance training on knee extensor strength. Med Sci Sports Exerc 1996;28:S191.

9 Rooney KJ, Herbert RD, Balnave RJ. Fatigue contributes to the strength training stimulus. Med Sci Sports Exerc 1994;26:1 160-4.

10 Schott J, McCully K, Rutherford OM. The role of metabolites in strength training. II. Short versus long isometric contractions. Eur J Appl Physiol 1995; 71:337-41.

11 Shinohara M, Kouzaki M, Yoshihisa T, et al. Efficacy of tourniquet ischemia for strength training with low resistance. Eur J Appl Physio 1998;77:189-91.

12 Welsh L, Rutherford OM. Effects of isometric strength training on quadriceps muscle properties in over 55 year olds. Eur J Appl Physiol 1996;72:219-23

13 Folland JP, Irish C, Roberts JC, et al. Fatigue is not essential in strength training. J Physiol (Lond) 1998;506:102-3P.

14 Parker DF, Round JM, Sacco P, et al. A cross-sectional survey of upper and lower limb strength in boys and girls during childhood and adolescence. Ann Hum Biol 1990;17:199-211.

15 Komi PV. How important is the neural drive for strength and power development in human skeletal muscle. In: Saltin B, ed. Biochemistry of exercise VI. Champaign, IL: Human Kinetics Publishers, 1986:515-30.

16 Gibala MJ, MacDougall JD, Tarnopolsky MA, et al. Changes in human muscle ultrastructure and force production after acute resistance exercise. J Appl Physiol 1995;78:702-8.

17 Newham DJ, Jones DA, Clarkson PM. Repeated high-force eccentric exercise: effects on muscle pain and damage. J Appl Physiol 1987;63:1381-6.

18 Folland JP, Copeman E, Chong J, et al. Acute muscle damage as a stimulus for training-induced gains in strength. Med Sci Sports Exerc 2002; in press.

19 Ciriello VM, Holden WL, Evans WJ. The effects of two isokinetic training regimens on muscle strength and fibre composition. In: Knuttgen HG, Vogel JA, Poortmans JR, eds. Biochemistry of exercise IV. Champaign, IL: Human Kinetics Publishers, 1983:787-93.

20 Narici MV, Roi GS, Landoni L, et al. Changes in force, cross sectional area and neural activation during strength training and detraining of the human quadriceps. Eur J Appl Physiol 1989;59:310-19.

21 Thomis MA, Beunen GP, Maes HH, et al. Strength training: importance of genetic factors. Med Sci Sports Exerc 1998;30:724-31.

22 Folland JP, Leach $B$, Little T, et al. Angiotensin converting enzyme genotype affects the response of skeletal muscle to functional overload. Exp Physiol 2000;85:575-9.

23 Graves JE, Pollock ML, Jones $A E$, et al. Specificity of limited range of motion variable resistance training. Med Sci Sports Exerc 1989;21:84-9.

24 Kanehisa $\mathbf{H}$, Miyashita $M$. Specificity of velocity in strength training. Eur J Appl Physiol 1983;52:104-6.

25 Caiozzo VJ, Perrine JR, Edgerton VR. Training induced alterations of the in-vivo force-velocity relationship of human muscle. J Appl Physiol $1981 ; 51: 750-4$ 


\section{COMMENTARY}

This paper has a useful message for sports medicine practitioners. Adherence to traditional training programmes for increasing muscle strength may be adversely affected by the level of pain and discomfort felt during performance of the training itself. The paper shows that for normal dynamic training of a large muscle group (quadriceps), the accumulation of metabolites-typically associated with the sensation of pain during exercise-is not a necessary characteristic of training conducted to increase muscle strength. The use of large rest periods between each repetition did not limit the gains in strength achieved compared with traditional sets of 10 repetitions. Therefore effective strength training can be conducted that produces less localised fatigue and muscle pain than traditional protocols. Adherence to training may be improved as a result of the reduced sensation of fatigue.

\section{A Williams}

Department of Sport, Health and Exercise, Staffordshire University, Leek Road, Stoke on Trent ST4 2DF, UK; a.williams@staffs.ac.uk

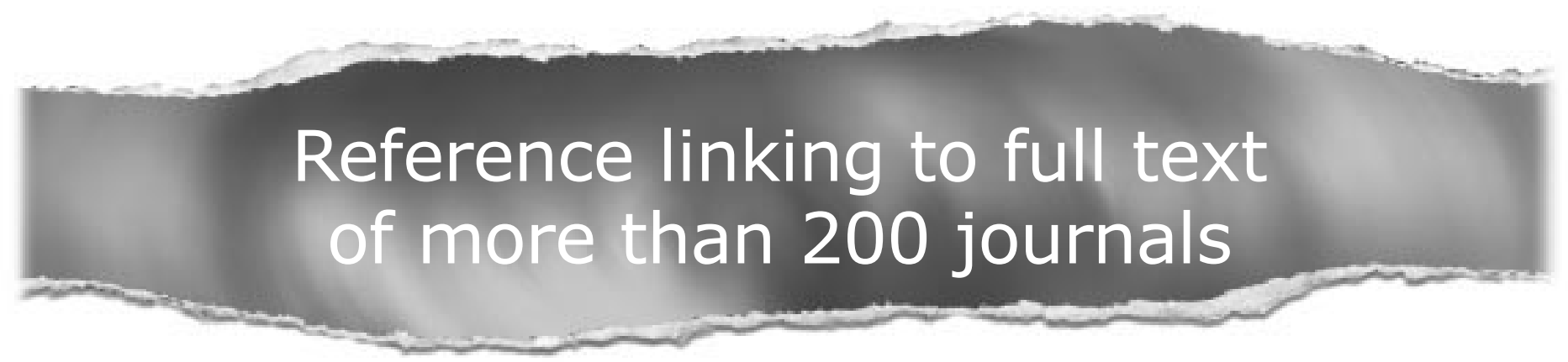

\section{Toll free links}

You can access the FULL TEXT of articles cited in the British Journal of Sports Medicine online if the citation is to one of the more than 200 journals hosted by HighWire (http://highwire.stanford.edu) without a subscription to that journal. There are also direct links from references to the Medline abstract for other titles.

\section{www.bjsportmed.com}

